\title{
Hyperviscosity syndrome - a case report
}

\begin{abstract}
Hyperviscosity syndrome (HVS) is a life-threatening complication. The clinical manifestations include a variety of symptoms like visual symptoms, altered mental status, stroke or congestive heart failure. Prompt treatment is needed to avoid progression to multisystem organ failure. We report a case of a 73-year-old man with a 3-months history of headaches and altered mental status. His neurological exam showed symmetrical decreased pain, thermal and tactile sensitivity in the upper and lower limbs and symmetrical decreased muscle strength in the lower limbs. His eye exam showed retinal hemorrhages and dilated retinal veins. His blood counts showed anemia, increased C-reactive protein, sedimentation rate and serum viscosity. He had an elevated immunoglobulin $\mathrm{M}$ and serum immunofixation revealed Ig M-kappa paraprotein. The bone marrow aspiration and biopsy revealed a Waldenström Macroglobulinemia. He was diagnosed with HVS and was treated with plasmapheresis, chemotherapy and fluids.HVS diagnosis requires a high degree of clinical suspicion, andclinicians should be aware of suggestive clinical and laboratory findings.
\end{abstract}

Keywords: waldenström's macroglobulinemia, hyperviscosity syndrome, plasmapheresis, paraproteinaemia, oncologic emergencies
Volume 3 Issue 4 - 2019

\section{Ana F Costa, Fábio Almeida, Ana F Batista, Joana D Antunes, Teresa Alfaiate, Fernando Ferraz,Amélia Pereira}

Internal Medicine, Hospital Distrital da Figueira da Foz, Portuga

Correspondence: Ana F Costa, Internal Medicine, Hospital Distrital da Figueira da Foz,Av. 12 de Julho 275, 3094-00I Gala, Figueira da Foz, Portugal,Tel 0035233402000 ,

Email asf.costa@hotmail.com

Received: July 22, 2019 | Published: August 30, 2019
Abbreviations: HVS, hyperviscosity syndrome; Ig, immunoglobulin; WM, waldenström's macroglobulinemia

\section{Introduction}

Hyperviscosity syndrome (HVS) refers to an increase in serum viscosity secondary to circulating proteins. It can be a result of an hyperproliferation of blood components such as seen en polycythemia and acute leukemias or a consequence of an increase in immunoglobulins. ${ }^{1,2}$ HVS is most common in patients with Waldenström's macroglobulinemia (WM), followed by multiple myeloma and leukemia., ${ }^{2,3}$ The clinical manifestations of HVS include a variety of symptoms like visual symptoms, altered mental status, stroke or congestive heart failure. The diagnosis of HVS should be suspected if it is presented the triad of mucosal or skin bleeding, visual abnormalities and neurologic deficits. ${ }^{3,4}$ Prompt treatment is needed to avoid progression to multisystem organ failure. The diagnosis is made by clinical and laboratory findings, like increased serum protein levels and increased serum viscosity. Management comprises supportive treatment and plasmapheresis. ${ }^{2,4}$ We report a case of a patient with HVS as a presentation of WM.

\section{Case report}

A 73-year-old man presented to our emergency department with a 3-months history of headaches and altered mental status (disorientation, agitation and inappropriate behavior). His wife also referred decreased sensitivity of the extremities of the upper limbs with burns in the fingers and progressive loss of autonomy. He denied fever, loss of weight, anorexia, nocturnal hyperhidrosis, hallucinations, seizures, thoracic and abdominal pain. He also denied intake of alcohol, tobacco, illicit drugs or herbal products. He had a history of auricular fibrillation, asbestos-related interstitial lung disease and benign prostatic hyperplasia. He was taking folic acid $5 \mathrm{mg}$ once daily, iron $329.7 \mathrm{mg}$ once daily, carvedilol $6.25 \mathrm{mg}$ twice daily, apixaban $5 \mathrm{mg}$ twice daily, furosemide $40 \mathrm{mg}$ twice daily, quetiapine $25 \mathrm{mg}$ once daily and lorazepam $1 \mathrm{mg}$ once daily. In the general examination, he was disoriented in time and space with a repetitive speech. He had burns and necrotic lesions on his fingers and toes. (Figure 1) The vital signs were normal, except for a tachycardia of $118 \mathrm{bpm}$. His physical examination revealed that he was pale, had cardiac arrhythmia and peripheral edemas. He had no palpable hepatomegaly, splenomegaly or lymphadenopathy. His neurological exam showed symmetrical decreased pain, thermal and tactile sensitivity in the upper and lower limbs and symmetrical decreased muscle strength in the lower limbs (grade 3/5).

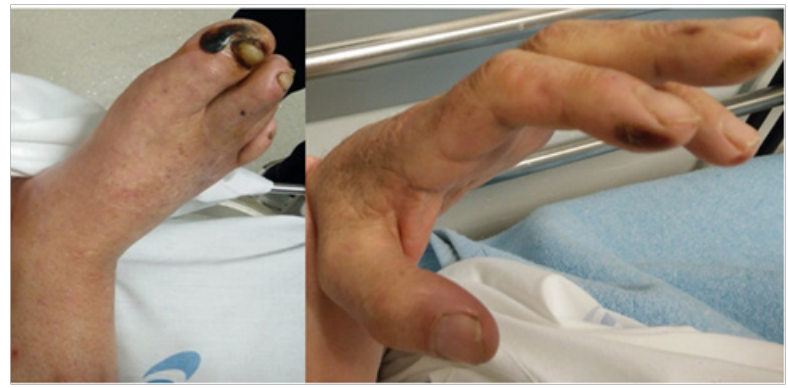

Figure I Necrotic lesions on toes and finger burns.

His blood counts showed anemia (hemoglobin $8.6 \mathrm{~g} / \mathrm{dL}$ ) and normal leukocyte and platelet levels. His laboratory investigations showed normal liver and renal enzymes, elevated C-reactive protein $(58.44$ $\mathrm{mg} / \mathrm{L}$, reference value $<5 \mathrm{mg} / \mathrm{L})$ and elevated sedimentation rate $(121$ $\mathrm{mm} / \mathrm{h})$. He had elevated folic acid $(>20 \mathrm{ng} / \mathrm{mL})$ and B12 vitamin (>2000 pmol/L) with low iron (36 ug/dL, reference value: 61-157 ug/ $\mathrm{dL})$ and normal ferritin and transferrin values. His thyroid tests were normal and his proBNP was high $(3285 \mathrm{pg} / \mathrm{mL}$, reference value $<300$ 
$\mathrm{pg} / \mathrm{mL})$. His total proteins were normal $(7.6 \mathrm{~g} / \mathrm{L})$ with low albumin $(2.9 \mathrm{~g} / \mathrm{dL}$, reference value: $3.4-4.8 \mathrm{~g} / \mathrm{dL})$ and high $\beta_{2}$-microglobulin $(5150 \mathrm{ug} / \mathrm{L}$, reference value: $800-2200 \mathrm{ug} / \mathrm{L})$. He had an elevated immunoglobulin M (IgM) $4227 \mathrm{mg} / \mathrm{dL}$ (reference value: $40-230 \mathrm{mg} /$ dL) with low $\operatorname{IgA}(63 \mathrm{mg} / \mathrm{dL}$, reference value: $70-400 \mathrm{mg} / \mathrm{dL})$ and $\operatorname{IgG}(507 \mathrm{mg} / \mathrm{dL}$, reference value: $700-1600 \mathrm{mg} / \mathrm{dL})$. His urinalysis showed more than 20 leukocytes per $\mathrm{uL}$. His chest $\mathrm{X}$ ray revealed an enlarged heart silhouette, bilateral opacities and pleural calcifications compatible with asbestosis. (Figure 2) The cranial computerized tomography (CT) scan was normal for his age.

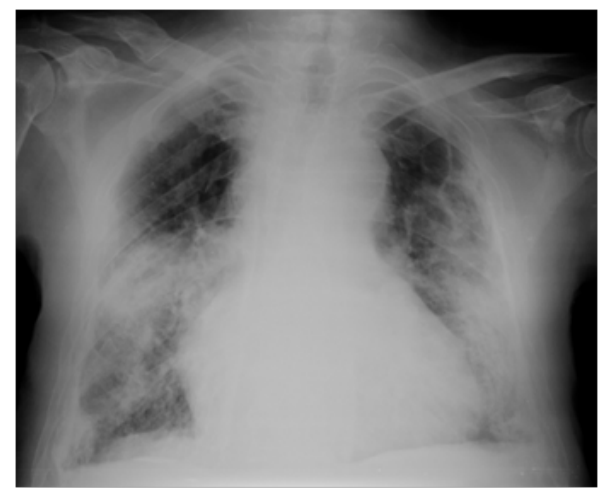

Figure 2 Chest $X$ - ray showing an enlarged heart silhouette, bilateral opacities and pleural calcifications.

The patient was hospitalized in the Internal Medicine ward with the diagnoses of urinary tract infection, decompensated heart failure and paraproteinaemia. During the hospitalization the paraproteinaemia work-up started. The eye exam showed retinal hemorrhages and dilated retinal veins. The serum viscosity was elevated $(2.2 \mathrm{cP}$, reference value $<1.8 \mathrm{cP}$ ). Serum immunofixation revealed IgM-kappa paraprotein and urinary immunofixation was normal. The patient had proteinuria with $1.89 \mathrm{~g} / 24 \mathrm{~h}$. The bone marrow aspiration and biopsy revealed an infiltration by small $\mathrm{CD} 20+$ lymphocytes in a nodular pattern and the genetic studies showed a positive mutation in MYD88 L265P. The abdominal ultrasonography showed a small hepatomegaly with a normal spleen. The patient was diagnosed with HVS caused by a WM and was transferred for the Hematology ward of the Hospital of University of Coimbra. He started treatment with plasmapheresis, chemotherapy and fluid therapy with an initial good response. He died after 6 months of treatment with an acute infection.

\section{Discussion}

$\mathrm{WM}$ is a rare B-cell disorder, characterized by over production of monoclonal IgM, with the median age at diagnosis of 70 years old. Many patients have constitutional symptoms (fevers, night sweats, weight loss, astenia), but substantial number of patients can be asymptomatic. The neuropathy is tipically sensory, bilateral and symmetrical and can evolve to muscle weakness. ${ }^{1,5}$ The diagnosis is based in the results of bone marrow biopsy and serum protein electrophoresis with immunofixation. ${ }^{5,6} \mathrm{HVS}$ is found in 10 to $30 \%$ of patients with WM and can be the initial manifestation. ${ }^{2,3} \operatorname{IgM}$ is a large molecular structure secreted as a pentamer and is found mainly in the intravascular structures. Its rich carbohydrate structure allows it to form aggregates, and its cationic nature decreases the repulsive forces of anionic red blood cells which leads to HVS. ${ }^{1,4}$ The diagnosis of HVS is based in clinical and laboratorial findings (elevated serum viscosity and serum protein levels). The levels of serum viscosity are related to the severity of clinical symptoms. Fundoscopic examination is important because can visualize a patognmonic sign of HVS - the sausage-shaped veins. Other ocular alterations are hemorrhagic and exsudative findings and retinal vein oclusions. ${ }^{2,4,7}$

After the diagnosis prompt therapy of HVS can prevent lifethreatening complications. Supportive treatment is based in intravenous fluids to prevent volume depletion. The primary treatment to revert the HVS complications is plasmapheresis and can reduce serum viscosity by 20 to $30 \%$ per session. The definitive treatment is the treatment of the underlying cause and it is chemotherapy in most cases. ${ }^{2,4}$ In this case, the patient had peripheral neuropathy, retinal hemorrhages and dilated retinal veins associated to necrotic lesions on the extremities. These findings are suggestive of HVS and the association of the diagnosis of WM with an increased serum viscosity made the diagnosis of HVS. The patient was treated with supportive treatment and plasmapheresis for HVS and with chemotherapy for $\mathrm{WM}$ as recommended by literature.

\section{Conclusion}

In conclusion, the HVS diagnosis requires a high degree of clinical suspicion, however the combination of clinical findings like mucosal or skin bleeding, visual abnormalities or neurologic deficitsand laboratory evidence of increased serum protein levels should raise the suspection.

\section{Acknowledgments}

The authors would like to thank the availability of the hematologists at the Coimbra Hospital.

\section{Conflicts of interest}

The author declares there is no conflict of interest.

\section{References}

1. Choi RY, Jacoby R, Shakoor A. Multimodality ocular imaging in a case report of Hyperviscosity syndrome associated with lymphoplasmacytic leukemia: the images tell the story. Retin Cases Brief Rep. 2019;13(3):238240 .

2. Khan UA, Shanholtz CB, McCurdy MT. Oncologic Mechanical Emergencies. Hematol Oncol Clin North Am. 2017;31(6):927-940.

3. Higdon ML, Atkinson CJ, Lawrence KV. Oncologic Emergencies: Recognition and Initial Management. Am Fam Physician. 2018;97(11):741-748.

4. Dumas G, Merceron S, Zafrani L, et al. Syndrome d'hyperviscosité plasmatique. La Revue de Médecine Interne. 2015:36(9):588-595.

5. Castillo JJ, Treon SP. Initial Evaluation of the Patient with Waldenström Macroglobulinemia. Hematol Oncol Clin North Am. 2018;32(5):811-820.

6. Caimi G, Carlisi M, Urso C, et al. Clinical disorders responsible for plasma hyperviscosity and skin complications. Eur J Intern Med. 2017;42:24-28.

7. Rajagopal R, Apte RS. Seeing through thick and through thin: Retinal manifestations of thrombophilic and hyperviscosity syndromes. Surv Ophthalmol. 2016;61(2):236-247. 